\title{
LA ALFABETIZACIÓN DIGITAL DEL DOCENTE UNIVERSITARIO EN EL ESPACIO EUROPEO DE EDUCACIÓN SUPERIOR
}

THE DIGITAL LITERACY OF UNIVERSITY PROFESSOR IN THE EUROPEAN HIGHER EDUCATION SPACE

\author{
Verónica Marín Díaz; vmarin@uco.es \\ Universidad de Córdoba \\ Ana I. Vázquez Martínez; aisabel@us.es \\ M미 del Carmen Llorente Cejudo; karen@us.es \\ Julio Cabero Almenara; cabero@us.es \\ Universidad de Sevilla
}

\section{RESUMEN}

La revolución tecnológica que en momento actual vivimos junto con la implantación del Espacio Europeo de Educación Superior, ha puesto de manifiesto la necesidad de una formación digital tanto en docentes. Para ello, se hace necesario que los docentes, en primera instancia, incorporen tanto a su vocabulario como a su metodología de aula la variable tecnológica. Desde estas páginas queremos hacer una llamada de atención a la reflexión sobre la necesidad de una formación digital en los docentes de cara al desarrollo del Espacio Europeo de Educación Superior coherente con la realidad social actual.

PALABRAS CLAVE: Universidad, Espacio Europeo de Educación Superior, Formación del profesor universitario, alfabetización digital,

\begin{abstract}
The technological revolution that the moment we live with the implementation of the European Higher Education has highlighted the need for computer training in teachers. To do this, it's necessary that the teachers in the first instance, incorporating both vocabulary and the methodology of classroom the technology variable. From this pages we want to do a clarion call to reflection on the need for digital literacy among teachers towards the development to de European Higher Education consistent with current social reality.
\end{abstract}

KEYWORDS: University, European Higher Education, digital training university teachers, digital literacy. 


\section{INTRODUCCIÓN}

El inicio del siglo XXI nos ha traído, entre otros aspectos, una nueva forma de ver, entender y sentir la docencia universitaria. La realidad de la implantación del Espacio Europeo de Educación Superior (EEES) ha supuesto la redefinición de las metodologías de aula así como la forma de entender y comprender los procesos de enseñanza-aprendizaje, que se deben construir desde esta nueva perspectiva. Los alumnos universitarios toman las riendas de su propio aprendizaje, son aprendices activos, críticos y autónomos. El profesorado, por su parte, toma el rol de guía en el proceso de búsqueda, selección y análisis de la información que la red proporciona a los estudiantes durante su ilustración.

Un ejemplo de esa preocupación que las universidades tienen por la capacitación digital o tecnológica de sus estudiantes, cuando finalicen los nuevos planes de estudio, lo encontramos en la solicitud de verificación de los títulos de grado por parte de la Universidad de Córdoba, quien considera de manera significativa el perfeccionamiento en el uso de las tecnologías de la información y la comunicación (TIC), es por ello que recoge como competencia universidad 2 (CU2): Conocer y perfeccionar el nivel de usuario en el ámbito de las TIC (Solicitud para la verificación de los Títulos de Grado, UCO, 2009, p. 39).

En consecuencia, si los estudiantes deben finalizar los estudios de grado con una formación digital, es necesario que a los docentes se les provea de ella.

Como resultado de esta situación nos encontramos un profesorado que, por un lado, ha de hacer frente a unos planes de estudio que parten del conocimiento de las TIC que los universitarios noveles han adquirido en niveles educativos inferiores, y por otro de unas habilidades y destrezas tecnológicas que deben saber responder a las demandas que la sociedad va a lanzar, o está lanzando ya, a las instituciones de educación superior en cuanto a la formación tecnológica de los futuros trabajadores.

El profesorado hoy se encuentra con una realidad universitaria que es compleja, en lo que se refiere a este tema. La literatura en torno a las generaciones que hoy pueblan las universidades (Boschman, 2006) los dibujan como competentes digitalmente hablando, sin embargo estudios realizados recientemente (Bullón, Cabero, Llorente, Machuca, Machuca y Marín, 2009; Cabero, Llorente y Puente, 2009; Marín y Cabero, 2010; Marín y Maldonado, 2011; Marín y Reche, 2011) han demostrado que los estudiantes universitarios, no han desarrollado la competencia digital de forma óptima en su formación previa a la universitaria. Si a esto se le añaden las exigencias que la implantación del Espacio Europeo 
de Educación Superior están presentando, nos encontramos a un profesor que debe saber, entre otros aspectos a:

- "Estimular el aprendizaje significativo y relevante de contenidos y de métodos de cada ámbito del saber.

- Estimular el desarrollo de actitudes de respeto y compromisos de la complejidad y diversidad de la realidad en cada ámbito del conocimiento.

- Fomentar el desarrollo de actitudes de curiosidad intelectual, búsqueda, duda, interrogación, indagación, experimentación, contraste, falsación, iniciativa y creatividad.

- Promover el desarrollo de capacidades y hábitos de estudio de trabajo cooperativo, de transferencia del conocimiento y de resolución de problemas prácticos" (CIDUA, 2005: 28).

Como señalan Solvberg, Rismark y Haaland (2009), la motivación del docente es crucial para la incorporación de las TIC al aula y, en consecuencia, esta se transmitirá al alumnado provocando una dinámica de aula más acorde a los nuevos roles que ambos deben asumir con el nuevo escenario didáctico que la implantación del EEES supone. La formación del profesorado para algunos autores (Northfield y Gunstone 1997, citado por De Vicente 2000) tiene dos propósitos:

- Ayudar a que aprenda y explique los conocimientos adquiridos sobre procesos de enseñanza-aprendizaje.

- Aprender a equilibrar el contexto y el currículum con la mejora de la enseñanza y el aprendizaje.

Junto a estos dos propósitos ahora debemos incluir la variable TIC, lo cual implica una formación específica en torno a ellas, lo cual entraña una redefinición de los roles del docente, de los estudiantes y de la propia institución universitaria.

\section{DE LA ALFABETIZACIÓN DIGITAL DEL PROFESOR UNIVERSITARIO}

La formación del profesorado universitario, desde nuestro punto de vista, debe englobar situaciones laborales diferentes, las cuales abarcarían tanto la reflexión sobre el trabajo realizado dentro del aula, como la realización de intercambios profesionales pasando por la experimentación de diversas situaciones y contextos. Todo ello presupone la puesta en 
práctica de una serie de experiencias de enseñanza-aprendizaje cuyo fin último será la creación y diseminación del conocimiento (Short, 2002).

La ausencia de una formación específica por parte del profesor universitario supone una circunstancia, que hoy en la mayoría de los países se ha convertido en una preocupación, principalmente por los hechos que rodean la nueva universidad que se quiere desarrollar a partir del curso académico 2010-2011. La formación de los docentes universitarios es vista como una herramienta además de como un arma estratégica para poder llevar a cabo una correcta reforma de los centros universitarios.

Hablar hoy de alfabetización digital supone entender que "no puede simplificarse en el conocimiento y manejo de las destrezas básicas lectoescritoras (hablar, escuchar, leer y escribir) en una sociedad actual" por lo que será "preciso considerar la alfabetización en medios o audiovisual, ya que los aprendizajes de nuestra época son en clave audiovisual" Aguaded y Pérez $(2006,69)$.

Partiendo de esta premisa debemos saber que hay varios tipos de alfabetizaciones, como señala Area, Gros \& Marzal, $(2008,73)$ : lecto-escritor, visual o icónico, numérico, musical o sonoro, audiovisual, informacional, y tecnológico o digital. Con respecto a las dos últimas podemos puntualizar que:

1) “Alfabetización en tecnologías y cultura digital (Conocer y saber utilizar el hardware de los distintos recursos tecnológicos. Conocer y saber manejar el software más relevante de los recursos digitales: procesadores de texto, imagen, navegadores, edición web,.... dominar las formas expresivas multimedia. Dominar las formas organizativas hipertextuales. Comunicarse y participar en redes sociales a través de tecnologías. Elaborar y difundir productos propios a través de tecnologías digitales).

2) Alfabetización informacional (Saber plantear estrategias y procesos para la resolución de problemas relacionados con la información. Saber buscar información en bases de datos o archivos de cualquier naturaleza (impresa, audiovisual, o digital) saber analizar e interpretar información presenta da a través de cualquier formato y/o tecnología. Saber producir información y difundirla a través de cualquier formato y/o tecnología".

Debemos ser conscientes, de que si aprender a ser docente (si aprender a enseñar) descansa en el acopio de forma sistemática de conocimientos, en la construcción y reconstrucción de creencias y en el desarrollo de aquellas técnicas válidas y necesarias para el buen funcionamiento de la docencia, será necesario que la alfabetización digital tanto inicial como permanente del profesorado universitario no se conviertan en terrenos abandonados por la implantación de paradigmas que alienen o reduzcan su formación, donde su objetivo principal sea la cualificación para un momento convergente que, paradójicamente, no es 
sentido por todos los miembros de la comunidad como un elemento positivo, ya que a veces al tener un sentido difuso, no presenta la realidad formativa-educativa en todo su esplendor.

Además de esto, se debe contemplar una segunda variable en el desarrollo del proceso formativo del docente y de la implantación del EEES, el conocimiento y la usabilidad que este le da u otorga a las TIC.

La tercera variable a tener en cuenta sería entender la comunicación, la creatividad y la gestión del cambio como tres elementos que marcarán el devenir de la educación universitaria de hoy, que deberán formar parte de la alfabetización digital de los docentes universitarios.

El informe Horizon (García, Peña-López, Johnson, Smith, Levine y Haywood, 2010 5), en su versión iberoamericana, señala que es un reto de este siglo XXI la alfabetización digital de docentes y estudiantes, reflejando que esa debe "convertirse en una aptitud esencial de la profesión docente. A pesar de que existe un consenso generalizado sobre su importancia, la formación en técnicas y aptitudes relacionadas con el ámbito digital sigue siendo una excepción en los programas de formación del profesorado. Las aptitudes y los estándares basados en herramientas y plataformas han demostrado ser algo efímeros, dado que la alfabetización digital no tiene tanto que ver con las herramientas como con el pensamiento: las competencias digitales tienen múltiples caras (tecnología, información, contenidos multimedia, identidad digital, etc.) y requieren ser afrontadas de forma comprehensiva... La formación de los estudiantes en el uso de los nuevos medios y lenguajes de comunicación audiovisual es un factor crítico. Los estudiantes necesitan nuevos conocimientos y aptitudes en el campo de la escritura y la comunicación, distintos de los que eran precisos hace tan sólo unos años. Cada vez más se hace necesario poseer conocimientos tecnológicos especializados para poder colaborar a escala global y ser capaces de comprender el contenido y el diseño de los nuevos medios. Por este motivo, deben integrarse en los planes de estudio las nuevas alfabetizaciones, así como su evaluación, algo que obliga a entender, en toda su extensión, el significado y el alcance de estas nuevas habilidades y competencias".

En consecuencia, la formación tecnológica o alfabetización digital implica que esta se caracterizará, como señala Maíz (2009), por tener un carácter crítico y reflexivo.

La alfabetización digital pretende ofrecer un marco conceptual para acceder, analizar, evaluar y crear mensajes en una variedad de formas, que vayan desde las impresas, pasando por los audiovisuales como los videos, hasta Internet y los multimedia. Por ello, los docentes 
deben asumir, entre otros, los siguientes principios, si desean incorporar las TIC a su actividad diaria, tanto docente como investigadora:

1) Todos los mensajes de los medios se construyen.

2) Los mensajes se construyen utilizando un lenguaje creativo que tiene sus propias reglas.

3) Distintas personas experimentan, entienden o interpretan el mismo mensaje de manera diferente.

4) Los medios tienen incorporados valores y puntos de vista.

5) Y los mensajes de medios se construyen para obtener ganancia y/o poder.

6) Al hilo de estos apuntes, la alfabetización digital debe proveer a los docentes de:

7) Un dominio en el manejo técnico de cada tecnología (conocimiento práctico del hardware y del software que emplea cada medio).

8) Proveer de un conjunto de conocimientos y habilidades específicas que les permitan buscar, seleccionar, analizar, comprender y recrear la enorme cantidad de información a la que se accede a través de las nuevas tecnologías.

9) Desarrollar un cúmulo de valores y actitudes hacia la tecnología, de modo que no se caiga ni en un posicionamiento tecnofóbico (es decir, que se las rechace sistemáticamente por considerarlas maléficas), ni en una actitud de aceptación acrítica y sumisa de las mismas.

10) Una visión en la que los medios y tecnologías se incorporen a su vida cotidiana, no sólo como recursos de ocio y consumo, sino también como entornos para expresión y comunicación con otros seres humanos.

11) Compartimos con Marín y Romero (2007) que la alfabetización digital del docente ha de caracterizarse por ser personalizada, flexible e interactiva, y que además debe potenciar todos los procesos de reflexión posibles (en la acción, para la acción y desde la acción), todo ello, claro está, desde una perspectiva donde se combine tanto la visión académica de las tecnologías como la práctica.

Debemos considerar que la inclusión de las TIC en la dinámica diaria de la institución universitaria en general y del desarrollo profesional de los docentes en particular viene cargada de una serie de ventajas, que Cabero ya en 2003 (161) sintetizaba en la "creación de entornos más flexibles para el aprendizaje, la eliminación de las barreras espacio-temporales entre docente y alumno, un incremento de las modalidades comunicativas, favorecer escenarios y entornos de carácter interactivo, el autoaprendizaje y el trabajo en grupo, salir de los muros del recinto educativo, ofrecer nuevas formas de tutoría y orientación del alumnado y favorecer la formación de carácter permanente". La falta de una alfabetización 
digital de los docentes implica que en estos momentos en los cuales se demanda una gran innovación en el currículo de los nuevos planes de estudio puestos en marcha, sea más bien escasa. En definitiva lo que se debe buscar, inicialmente, es un cambio en la concepción de la alfabetización tecnológica de los profesores.

Todo este proceso formativo, que dese estas líneas estamos demandando. implica que el profesorado lo tome como suyo, lo cual, además, entraña entender que esta se apoyará en el learning by doing alejada de la concepción del teaching by telling.

\section{PARA IR FINALIZANDO}

Una de las características de la denominada sociedad del conocimiento (Cabero, 2001), es que esta gira alrededor de las TIC, como elemento nuclear de las mismas, alcanzado su penetración a todos los sectores, desde los políticos hasta los económicos, desde los culturales hasta los educativos, convirtiéndose al mismo tiempo en un valor económico. Lankshear y Knobel (2008) nos recuerdan que hasta hace poco la presencia del concepto alfabetización en el ámbito de la educación formal era más bien escaso; hoy la realidad comienza a cambiar de prisma, pues empieza a haber una preocupación, tanto por los políticos universitarios como por los teóricos de la educación superior, porque los profesores estén actualizados en estos términos.

Como ya hemos señalado anteriormente, esta realidad es trasladada a las universidades como transmisoras de la formación que la sociedad demanda en sus trabajadores. La incorporación de la variable tecnológica y del desarrollo del EEES supone una reestructuración de toda la vida universitaria.

En lo que se refiere a los docentes, implica que estos tengan una nueva visión de los procesos de enseñanza aprendizaje, además de desarrollar una nueva forma de entender la didáctica universitaria. Pues, como vemos, las implicaciones que tiene la incorporación de la variable TIC a la universidad es imponente y, en consecuencia, las aulas universitarias deben, desde el binomio TIC-EEES, saber dar una respuesta acorde a tal realidad. Por tanto, esta relación implica que la inclusión de las TIC en la alfabetización o capacitación digital de los profesores universitarios se tenga en cuenta que estas han de:

- Ser entendidas como un espacio social de carácter horizontal.

- Ser espacios ricos en información.

- Ser espacios multifuncionales para la acción y la reflexión. 
- Facilitar tanto el aprendizaje autónomo como el colaborativo.

- Permitir la elaboración o remezcla de materiales educativos.

- Facilitar la creación de redes de aprendizaje

- Y desarrollar la competencia digital de la comunidad universitaria.

En definitiva. la capacitación digital del docente universitario se ha convertido en una variable independiente dentro de las políticas que hoy se están dibujando, tanto a nivel local como nacional e internacional. Solo así, los futuros profesionales que llevaran las riendas de la sociedad podrán hacer que esta marche de forma correcta en su progreso.

\section{REFERENCIAS BIBLIOGRÁFICAS}

AGUADED, J. I. Y PÉREZ, M. A. (2006). La educación en medios de comunicación como contexto educativo en un mundo globalizado. En J. Cabero (Coord.), Nuevas tecnologías aplicadas a la educación, (pp. 63-75). Madrid. McGraw-Hill,

AREA, M., GROS, B. Y MARZAL, M. (2008). Alfabetizaciones y tecnologías de la información y la comunicación. Madrid: Síntesis.

BOSCHMAN, J. (2006). Generación Einstein. Más listos, más rápidos y más sociables. Barcelona: Gestión 2000.

BULlón, P., CABERO, J., LLORENTE, Mạ C., MACHUCA, Mạ C., MACHUCA, G. Y MARÍN, V. (2009). Competencias tecnológicas del profesorado de la Facultad de Odontología de la Universidad de Sevilla. Sevilla: GID.

CABERO, J. (2001). Tecnología educativa. Diseño y utilización de medios en la enseñanza. Barcelona: Paidós.

CABERO, J. (2003). Las tecnologías de la comunicación, nuevo espacio para el encuentro de los pueblos iberoamericanos. Comunicar, 20, 159-167. Disponible en http://www.revistacomunicar.com/index.php?contenido=detalles\&numero=20\&articul $\underline{0=20-2003-23}$

CABERO, J., LLORENTE, M.C. Y PUENTES, A. (2009A). Alfabetización digital: un estudio en la pontificia Universidad Católica Madre y Maestra. Sevilla: Grupo de Investigación Didáctica.

CABERO, J., LEAL, F., ANDRÉS, F. Y LLORENTE, M. C. (2009B). Capacitación digital de los alumnos de la Unidad Académica Multidisciplinaria de Agronomía y Ciencias (Ciudad 
Victoria) de la Universidad Autónoma de Tamaulipas. Sevilla: Grupo de Investigación Didáctica.

CIDUA (2005). Informe sobre innovación de la docencia en las universidades andaluzas. Disponible en http://viceees.ujaen.es/files viceees/CIDUA.pdf.

GARCíA, I., PEÑA-LÓPEZ, I., JOHNSON, L., SMITH, R., LEVINE, A. Y HAYWOOD, K. (2010). Informe Horizon: Edición Iberoamericana 2010. Austin, Texas: The New Media Consortium.

LANKSHEAR, C. Y KNOBEL, M. (2008). Nuevos alfabetismos. Su práctica cotidiana y el aprendizaje en el aula. Madrid, Morata.

MAÍZ, I. (2009). Implicaciones educativas de herramientas tecnológicas de la web 2.0. En C. Castaño (coord.). Web 2.0. El uso de las web en la sociedad del conocimiento, (pp. 177195). Caracas: Universidad Metropolitana.

MARÍN, V. Y CABERO, C. (2010). Del conocimiento del estudiante universitario sobre las herramientas 2.0. Revista Anales de la Universidad Metropolitana, 10 (2), 51-74. Disponible en: http://dialnet.unirioja.es/servlet/articulo?codigo=3408817

MARÍN, V. Y MALDONADO, G.A. (2011). El alumnado universitario cordobés y la plataforma virtual Moodle. Pixel-Bit, Revista de Medios de Comunicación, 38, 121-128. Disponible en: http://www.sav.us.es/pixelbit/actual/9.pdf

MARÍN, V. \& ROMERO A. (2007). Las redes de comunicación para el aprendizaje y la formación docente universitaria. EDUTEC, Revista Electrónica de Tecnología Educativa, 23, 1-11. Disponible en http://edutec.rediris.es/Revelec2/revelec23/marin romero/marin romero.html.

MARÍN, V. Y RECHE, E. (2011). La alfabetización digital del alumnado que accede a la Universidad de Córdoba. EDUTEC, 35. Disponible en http://edutec.rediris.es/Revelec2/Revelec35/alfabetiazacion_digital_alumnado_univer sidad cordoba.html.

NORTHFIELD, J. Y GUNSTONE, R. (1997). Teacher education as a process of developing teacher knowledge. En J. Laughten \& T. Russell (eds.). Teaching about teaching purpose, passion and pedagogy in teacher education, (pp. 48-56). London: Falmer Press, Citado en De Vicente, P.S. (2000). Cultura e iniciación profesional del docente. En Lorenzo, M. \& colaboradores (coords.). Las organizaciones educativas en una sociedad neoliberal, (pp. 919-940). Granada: GEU y AREA,

SHORT, E.E. (2002). Knowledge and the educational funtions of a university designing the curriculum of higher education. Journal Curriculum Studies, 3 (22), 139-148. 
SOlVBERG, A. M., RISMARK, M. Y HAALAND, E. (2009). Teacher and technology in the making: developing didactic competence. Procedia Social and Behavioral Sciences,1, 2791-2794.

Para citar este artículo:

MARÍN, V., VÁZQUEZ, A.I., LLORENTE, M.C. \& CABERO, J. (2012). La alfabetización digital del docente universitario en el Espacio Europeo de Educación Superior. Edutec, Revista Electrónica de Tecnología Educativa, 39. Recuperado el dd/mm/aa de http://edutec.rediris.es/Revelec2/Revelec39/alfabetizacion digital docente universitario E EES.html 